\title{
Heart Rate Variability Predicts Treatment Outcome in Major Depression
}

\author{
Brandon Hage ${ }^{1}$, James Sinacore ${ }^{2,}$ Keri Heilman ${ }^{3}$, Stephen W. Porges \\ ${ }^{3,4}$, Angelos Halaris ${ }^{1^{*}}$ \\ ${ }^{1}$ Department of Psychiatry and Behavioral Neurosciences, Loyola University \\ Chicago, Stritch School of Medicine, USA; \\ ${ }^{2}$ Department of Public Health, Loyola University Chicago, Stritch School of \\ Medicine, USA; \\ ${ }^{3}$ Department of Psychiatry, University of North Carolina, Chapel Hill, NC, USA; \\ ${ }^{4}$ Kinsey Institute, Indiana University Bloomington, Indiana, USA.
}

*Correspondence: Angelos Halaris, MD, PhD, Email: ahalaris@lumc. edu.

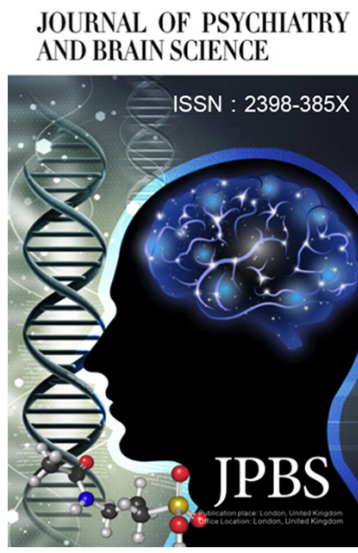

http://jpbs.qingres.com

\section{fOPEN ACCESS}

DOI: 10.20900/jpbs.20170017

Received: November 2, 2017

Accepted: November 2, 2017

Published: December 25, 2017

Copyright: $\odot 2017$ Cain et al. This is an open access article distributed under the terms of the Creative Commons Attribution License, which permits unrestricted use, distribution, and reproduction in any medium, provided the original author and source are credited.

\begin{abstract}
Aims: Autonomic nervous system (ANS) dysregulation is associated with various symptoms of depressive disorder. The beat-to-beat pattern of heart rate (Heart Rate Variability) (HRV) provides a noninvasive portal to ANS function through the quantification of periodic heart rate patterns. In this study we quantified two components of HRV: Respiratory Sinus Arrhythmia (RSA), and Low Frequency HRV (LFHRV). Both of these components have been extensively reported in studies of depression and have been at least partially associated with reduction in vagal nerve tone. We quantified RSA and LF-HRV in patients with Major Depressive Disorder (MDD) as measures of ANS regulation seeking to establish the utility of components of HRV as potential diagnostic and prognostic biomarkers for treatment outcome.
\end{abstract}

Methods: Sixty-six MDD patients were enrolled. In two separate and consecutively run studies they received either Escitalopram or Quetiapine fumarate ER over 12 weeks. Forty-one patients completed the studies. RSA and LF-HRV were assessed at pretreatment and end of study. Thirty-six healthy subjects served as controls. RSA and LFHRV were quantified using an algorithm that incorporates time and frequency domains to address the non-stationarity of the beat-to-beat heart rate pattern.

Results: No significant differences in baseline RSA or LF-HRV were found between MDD and healthy controls. However, baseline RSA and LF-HRV were significantly higher in treatment responders (InRSA = 
6.20$, InLF-HRV $=5.76)$ than non-responders (InRSA $=4.75$, InLF-HRV = 4.53) $(p=0.005, p=0.006)$. No significant changes in RSA or LF-HRV were detected over the course of either treatment.

Conclusions: Components of HRV may be predictive of antidepressant response in MDD patients.

Keywords: Escitalopram; Heart rate variability; Major depressive disorder; Quetiapine; Respiratory Sinus Arrhythmia; Low-frequency HRV

\section{INTRODUCTION}

Major Depressive Disorder (MDD) is a highly prevalent disorder worldwide. At any time, at least 8-10\% of the population of the United States is afflicted with this disorder ${ }^{[1]}$. By 2030, depression will be the number one contributor to the global disease burden ${ }^{[2]}$. A major pathophysiological mechanism believed to underlie MDD is dysregulation of Autonomic Nervous System (ANS) function ${ }^{[3]}$. Associated reduction in parasympathetic tone due to illness severity, recurrence and chronicity may lead to disinhibition of the body's inflammatory response as the immune system becomes overactive ${ }^{[4]}$. Vagal nerve efferent fibers regulate inflammation via the Cholinergic Anti-inflammatory Pathway (CAIP) ${ }^{[5}$, ${ }^{6]}$. We sought to determine whether ANS activity is dysregulated in depression by studying Heart Rate Variability (HRV) as a peripherally derived noninvasive measure of ANS activity. Additionally, we explored the relationship between antidepressant treatment response and HRV and whether ANS status, as assessed by HRV, can predict treatment outcome. Although HRV and its relationship to diagnosis of MDD has been studied in greater depth more recently ${ }^{[7-9]}$, HRV in relation to treatment outcome in MDD is largely unknown.

HRV is defined as the variation between heartbeats over a period of time; it involves input from the sympathetic and parasympathetic divisions of the ANS. Short duration recordings on an electrocardiogram (ECG) produce two primary patterns of oscillation ${ }^{[10]}$. One oscillation occurs within period between about 2 and 8 s (approximately one breath cycle in the general population), which corresponds to $0.120 .40 \mathrm{~Hz}$ (High frequency or HF-HRV). This frequency was selected to be consistent with range of breathing frequencies in adults ${ }^{[11]}$. The commonly used lower threshold of $0.15 \mathrm{~Hz}$, will miss sources of HRV associated with slow breaths. The frequency of this oscillation coincides with a physiological phenomenon known as Respiratory Sinus Arrhythmia (RSA), which is characterized by an oscillation in the beat-to-beat heart rate pattern that occurs at the frequency of spontaneous breathing. RSA has been used as a validated estimate of cardiac vagal tone ${ }^{[11,12]}$. The second oscillation (i.e., LF-HRV) occurs at a slower frequency with a periodicity between about 10 and 25s, which corresponds to $0.04-0.10 \mathrm{~Hz}$. Initially, it was assumed and reported that the sympathetic nervous system contributed to LF-HRV. However, recent studies have postulated that LF is a poor measure of sympathetic activity and, similar to RSA, is primarily mediated via the vagus nerve ${ }^{[13-16]}$. This latter point has been confirmed by experiments using administration of atropine, a vagal blocker, which blocked both oscillations ${ }^{[17]}$. Although we measured both components of HRV, given that substantial literature has validated RSA as an index of cardiac vagal tone ${ }^{[18]}$ and in view of the ambiguity in interpreting LF-HRV ${ }^{[19,20]}$, we have chosen to emphasize RSA as our primary index of ANS function.

HRV has been investigated in psychiatric populations, and decreased HRV has been associated with anxiety disorder, bipolar mania, schizophrenia, and MDD ${ }^{[21-23]}$. Finally, other investigators have associated reduced HRV with several symptoms of MDD, such as sadness and suicidality ${ }^{[24,25]}$.

The primary goal of this study was to determine whether HRV components would be reduced in MDD subjects and whether the assessed components could be associated with the diagnosis and treatment of MDD. An ancillary goal was to seek possible associations between autonomic dysfunction, as assessed with HRV, and the immunological pathophysiology of MDD described in the literature.

\section{METHODS}

\subsection{Study population}

The study was approved by the Institutional Review Board (IRB) of Loyola University Medical Center and was conducted according to the principles of the Declaration of Helsinki. Males and females 20-65 years of age who met DSM-IV criteria for MDD, first episode or recurrent type, who were otherwise physically healthy and mentally capable to give informed consent, were considered as candidates. Their index episode had to be of at 
least 1-month duration and they could not have had psychopharmacological treatment over the preceding four weeks. A minimum score of 18 on the 17-item Hamilton Depression Scale (HAMD17) was required for study eligibility. This score is used conventionally to indicate moderate severity of depressive symptomatology ${ }^{[26]}$. Other Axis I and II diagnoses, active suicidality, hypertension, dyslipidemia, diabetes mellitus, history of smoking or substance abuse in the preceding 6 months, and history of heart disease were exclusion criteria. Subjects had to be free of any source of active or chronic inflammation. Female subjects could not be pregnant, lactating, or taking oral contraceptives. Screening blood samples were obtained to determine complete blood count, complete metabolic panel, lipid profile, thyroid function and urinalysis (including pregnancy test). The presence of any clinically significant abnormalities excluded the prospective participant. Sixty-six MDD patients who met the inclusion/exclusion criteria and successfully completed the baseline evaluations were enrolled in one of the two consecutive treatment studies. Their demographic data is shown in Table 1.

Table 1. Demographic characteristics of MDD patients vs. Healthy Control subjects.

\begin{tabular}{llll}
\hline & MDD Subjects & Healthy Control Subjects & p/x2 value \\
\hline Study Participants & 41 & 36 & 0.48 \\
Age $( \pm$ SD) & $41.4(11.8)$ & $39.3(13.8)$ & 0.003 \\
BMI $( \pm$ SD) & $31.1(6.7)$ & $26.7(5.9)$ & 0.97 \\
Female & $63.4 \%$ & $63.9 \%$ & 0.047 \\
Caucasian & $53.7 \%$ & $75.0 \%$ & \\
\hline
\end{tabular}

\subsection{Healthy control subjects}

Eligible healthy control (HC) subjects were recruited by advertising and posting of IRB approved flyers. To determine eligibility, identical procedures were used as for the MDD group including a psychiatric diagnostic structured interview and routine laboratory tests. Main exclusion criteria were any medical, inflammatory or mental illness and substance use (the latter also amongst first degree relatives). Pregnant or lactating females were excluded. Their HAMD-17 and Beck Depression Inventory (BDI) scores had to be less than 5 . Thirty-six subjects were enrolled resulting in a ratio MDD/HC of about 2:1. Their demographic data is shown in Table 1.

\subsection{Study design}

Two screening visits (Screen-1 and -2) preceded all baseline measurements to determine study eligibility, obtain informed consent and allow for acclimation of the subject to the setting and the study team. The baseline visit followed, unless the mandatory four-week antidepressant washout period had to occur first. Patients were contacted by research team members to ensure their safety should they develop suicidal ideation. No such incident occurred throughout the duration of the study. Maintenance anti-anxiety and / or hypnotic medication was allowed to continue at the discretion of the clinician and only if the subject had been taking it for at least one month. No discontinuation was permitted throughout the study. Less that ten percent of the study population was maintained on such medication.

After the baseline assessments were completed, patients were started on either Escitalopram (Study-1) or Quetiapine fumarate ER (Study-2). Study-1 was undertaken in order to investigate the effect of Escitalopram monotherapy on select inflammation biomarkers ${ }^{[27]}$. Study-2 was undertaken in order to test the hypothesis that Quetiapine monotherapy would exert comparable antidepressant effectiveness as a conventional SSRI antidepressant (in preparation). Additionally, the effect of this atypical antipsychotic agent on select inflammation biomarkers was also tested. Escitalopram dosing was started at $10 \mathrm{mg} / \mathrm{day}$ and 
maintained in the range of $10-30 \mathrm{mg} /$ day. Quetiapine dosing was started at $25 \mathrm{mg} / \mathrm{day}$ and titrated at the discretion of the investigators up to a $300 \mathrm{mg} / \mathrm{day}$. Enrolled patients received no other form of therapy for the duration of the study. Blood draws and clinical assessments were performed at weeks $0,2,4,8$, and 12.

Patients were required to complete at least 8 weeks of active study participation and end-of-study assessments to be regarded as completers. Patients who chose to withdraw from the study on or after 8 weeks of treatment, and completed the battery of final assessment tests, had their results carried forward for data analysis.

\subsection{Collection of HRV data}

HRV was assessed at weeks 0,8 , and 12 using the SphygmoCor ${ }^{\circledR} \mathrm{CPVH}$ system. The subject reclined on the examination table, a three-lead ECG with a sampling rate of $1024 \mathrm{~Hz}$ was attached to the chest in the LEAD II configuration, and a rest period 1015 minutes followed before the ECG recording was started. The entire recording was obtained in the supine position. ECG data were collected over a 15-minute period to ensure consistency in data collection. Short-term HRV measurements (30 minutes or less) are stable over a significant period of time as compared to 24-hour measurements via a Holter monitor ${ }^{[28,29]}$.

\subsection{Inter-Beat-Interval editing and analysis}

Data collected for HRV quantification are subject to artifacts that are related to the function of the ECG. Components of HRV are calculated from a time series generated by timing the interval between sequential heartbeats (i.e., msec between sequential R-waves in the ECG) during a period of 10-15 minutes. This time series consists of several hundred values that correspond to individual interbeat-intervals (IBIs). Physiological mechanisms, both related and unrelated to RSA, can contribute to this time series by distorting the accuracy of the R-wave detection. Influences from a spurious decrease in $\mathrm{R}$ wave amplitude, a random abnormally large $T$ wave, single PACs/PVCs, or ECG signal quality disrupted by patient activity must be removed from the time series before the components of HRV can be reliably quantified.

To deal with potential anomalies through artifact or ventricular arrhythmia (both components of HRV are manifestations of atrial rhythms that represent the time course of the vagal influences on the sinoatrial node), a software package was used to correct for any artifacts ${ }^{[30]}$. Editing involved integer arithmetic to adjust the time series by adding IBIs when false invalid intervals occurred and dividing IBIs when $\mathrm{R}$-wave detections were missed. These decisions were guided by inspection of the ECG. In order to preserve an accurate representation of the neural regulation of the heart, data were only accepted if less than $5 \%$ of the data needed to be corrected.

After visual scanning and editing, the data were analyzed ${ }^{[31]}$ with CardioBatch. CardioBatch is the companion program to CardioEdit, which was used to quantify RSA and LF-HRV. The program incorporates algorithms that have been developed to extract oscillatory components in the nonstationary beat-to-beat heart rate pattern ${ }^{[32]}$. Fifteen minutes of ECG data were collected for each individual. Values for heart rate, RSA, and LF-HRV were calculated in sequential 30-second epochs and then averaged across the 15-minute period. RSA and LF-HRV values for each epoch were transformed to their natural logarithmic values to conform to the distributional requirements for parametric analyses ${ }^{[33,}$ 18]

\subsection{Measurement of inflammation biomarkers}

Blood was drawn between 9 and 10 am at baseline, and week 12 for MDD subjects. Whole blood was spun to separate plasma or serum and both were stored at -80 degrees centigrade until analyzed. Plasma samples were analyzed using a biochip immunoassay system from Randox Laboratories Ltd (Crumlin, UK). The Biochip Array technology was used to simultaneously detect the quantity of multiple analytes (cytokines, chemokines, and growth factors) from a single plasma sample. The Randox Biochip contains a selection of discrete test regions for antibodies specific to certain cytokines and growth factors. A sandwich chemiluminescent immunoassay was used for the cytokine array. Each test region generates a light signal that is detected by digital imaging technology (charged couple detector). The concentration of each analyte present is calculated from a calibration curve. Each calibration began at 0 $\mathrm{pg} / \mathrm{ml}$. According to the manufacturer, the inter-assay and intra-assay variation is less than $10 \%$. We validated this Biochip technology for blood samples in several previous studies. 


\subsection{Statistical analyses}

Consistent with the literature that documents RSA and LF-HRV decreases with advancing age ${ }^{[34,35]}$, both the MDD and HC groups exhibited a significant negative relationship between age and both components of HRV. Additionally, the MDD group had a significantly higher BMI than the $\mathrm{HC}$ group and less representation of Caucasian participants than the control group. Given the potential for these variables to affect our dependent variables, analyses of covariance (ANCOVA) were used to control for potential effects of age, sex, BMI and ethnicity for all analyses of RSA and LF-HRV (Table 1). Repeated measures ANCOVA were used to evaluate potential changes in RSA and LF-HRV from baseline to week 12.

Treatment response in study completers was classified as a) no response to treatment (less than $50 \%$ reduction in HAM-D score from baseline); b) partial response to treatment (greater than $50 \%$ reduction but end-of-study HAM-D score greater than 7 ); c) remission (end-of-study HAM-D score of less than 7). It is generally accepted that MDD patients who have a partial response to antidepressant treatment are at much higher risk of experiencing physical and mental dysfunction in comparison to patients who achieve remission ${ }^{[36]}$. For purposes of these analyses, no response and partial response were grouped into the non-response category. ANCOVA compared baseline RSA and LF-HRV between treatment responders and non-responders.

To test for group differences in baseline RSA and LF-HRV, based on psychiatric variables within the MDD group, ANCOVA analyses were conducted separately for the following group variables: previous psychiatric medication use, previous occurrence of depressive episodes, and length of current depressive episode (partitioned into either one of three groups: one to six months, six months to one year, or greater than one year).

Level of significance was set at $p \leq 0.05$.

\section{RESULTS}

Collapsed across groups, baseline RSA and LFHRV were significantly correlated, $r(77)=0.70, p$ $<0.0005$. Significant correlations occurred within groups as well, MDD: $r(41)=0.72, p<0.0005$; $\mathrm{HC}$ : $r(36)=0.70, p<0.0005$.

Univariate ANCOVA was used to evaluate group differences in baseline RSA and LF-HRV between MDD patients $(n=41)$ and HC subjects $(n=36)$. Baseline RSA did not distinguish MDD patients (M $=5.73, \mathrm{SE}=0.20)$ from $\mathrm{HC}$ subjects $(\mathrm{M}=5.65$, $\mathrm{SE}$ $=0.22$ ) (Fig. 1). Similarly, baseline LF-HRV did not distinguish MDD patients $(M=5.37, S E=0.17)$ from $\mathrm{HC}$ subjects $(\mathrm{M}=5.50, \mathrm{SE}=0.17)$ (Data not shown).

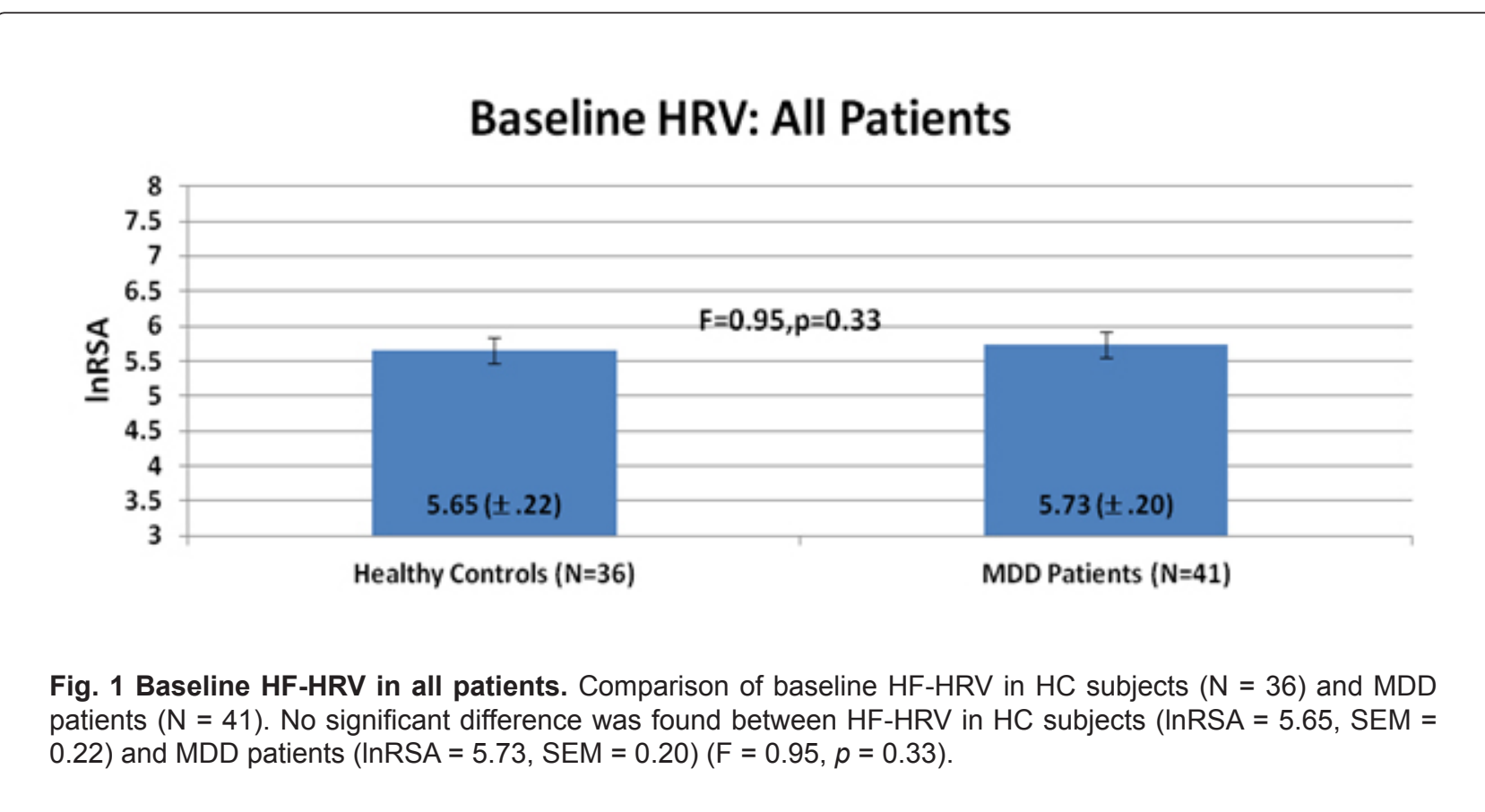


Repeated measures ANCOVA were used to evaluate change in RSA and LF-HRV from baseline through completion of the study, by treatment group (Escitalopram $n=22$; Quetiapine $n=12$ ). For RSA, there were no condition effects, no interaction, and no treatment group effect (Fig. 2). Similarly, for LF$\mathrm{HRV}$, there were no condition effects, no interaction, and no treatment group effect (Data not shown).

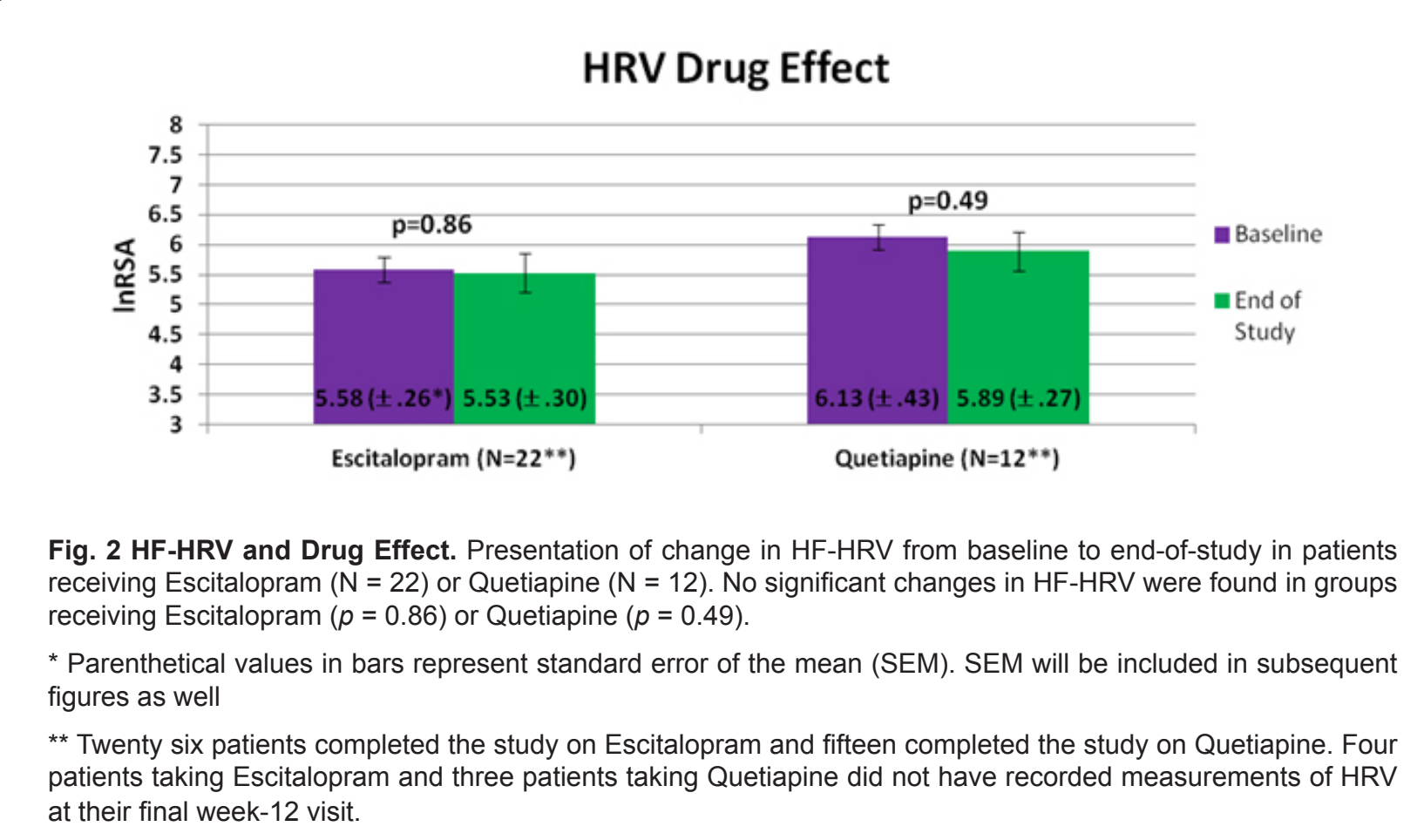

Collapsed across treatment groups, the MDD group was partitioned into treatment responders ( $\mathrm{n}$ $=28$ ) and non-responders $(n=13)$. Baseline RSA for treatment responders $(M=6.18, S E=.22)$ was significantly higher than non-responders $(M=4.78$, SE $=0.29), F(1,35)=9.05, p<0.005$, Cohen's $d=$
1.26 (Fig. 3). The pattern of responding within each drug group was similar (Fig. 4). Baseline LF-HRV for treatment responders $(M=5.76, S E=0.14)$ was significantly higher than non-responders $(M=4.53$, SE $=0.36), F(1,35)=8.44, p<0.006$, Cohen's $d=$ 1.32 (Data not shown).

\section{Baseline HRV: Treatment Response}

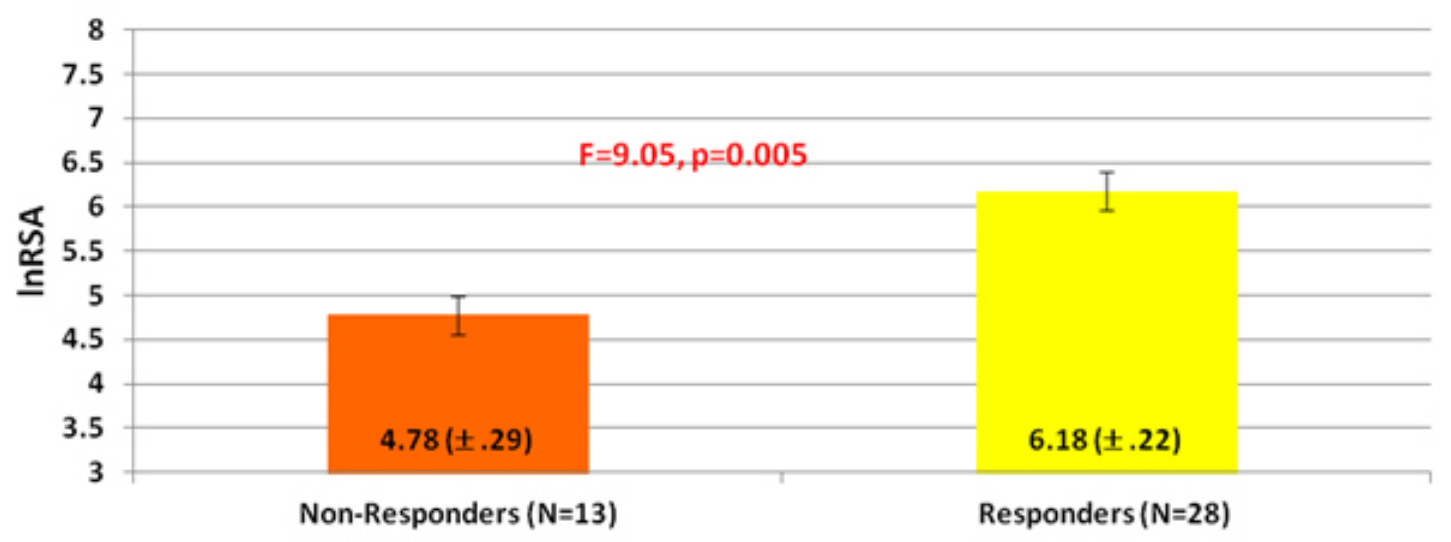

Fig. 3 Baseline HF-HRV and treatment response overall. Comparison of baseline HF-HRV in MDD treatment responders $(N=28)$ versus MDD treatment non-responders $(N=13)$. Baseline HF-HRV was significantly higher in treatment responders $(\operatorname{InRSA}=6.18$, SEM $=0.22)$ than in treatment non-responders $(\operatorname{InRSA}=4.78, \mathrm{SEM}=$ 0.29) $(\mathrm{F}=9.05, p=0.005)$. 
Baseline HRV: Treatment

Response to Escitalopram

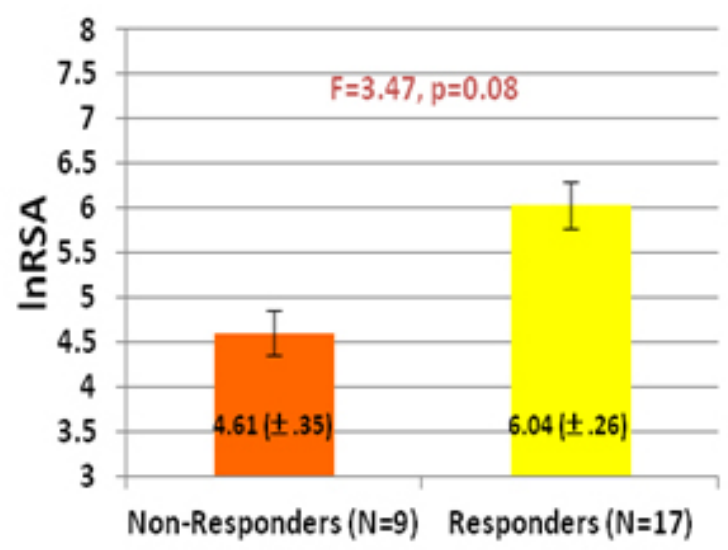

Baseline HRV: Treatment Response to Quetiapine

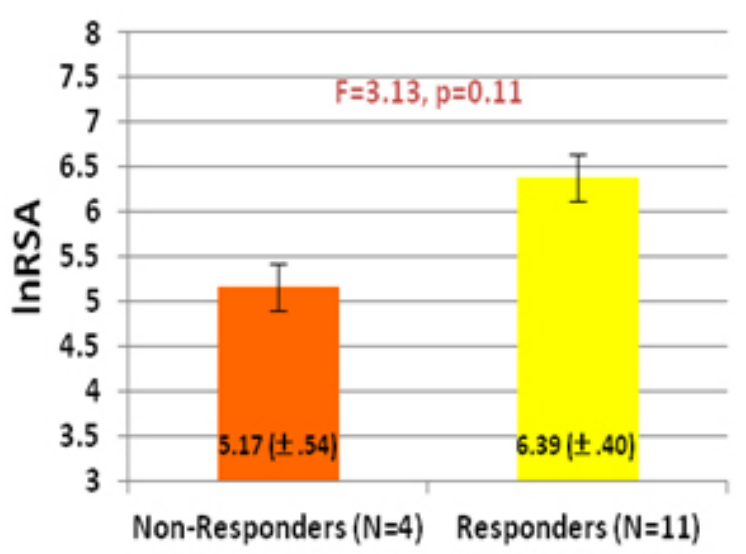

Fig. 4 Baseline HF-HRV and treatment response to Escitalopram or Quetiapine. Comparisons of baseline HF-HRV in Escitalopram responders $(\mathrm{N}=17)$ and non-responders $(\mathrm{N}=9)$, and Quetiapine responders $(\mathrm{N}=$ 11 ) and non-responders $(N=4)$. The difference between Escitalopram responders $(\operatorname{InRSA}=6.04, \mathrm{SEM}=0.26)$ and non-responders $(\operatorname{InRSA}=4.61, \mathrm{SEM}=0.35)$ was trending toward significance $(\mathrm{F}=3.46, p=0.08)$. The difference between Quetiapine responders $(\operatorname{InRSA}=6.39, \mathrm{SEM}=0.40)$ and non-responders $(\operatorname{InRSA}=5.17$, SEM $=0.54)$ was trending toward significance $(F=3.13, p=0.11)$.

The MDD group was partitioned based on history of prior use of psychiatric medication $(n=$ $24)$ or no prior psychiatric medication use $(n=15)$. Baseline RSA for patients with a history of prior use of psychiatric medication use $(\mathrm{M}=5.25, \mathrm{SE}=0.24)$ was significantly lower than patients with no prior psychiatric medication use $(\mathrm{M}=6.47, \mathrm{SE}=0.31), \mathrm{F}$ $(1,33)=7.33, p<0.01$, Cohen's $d=1.05$ (Fig. 5). However, differences in baseline LF-HRV were nonsignificant.

\section{Baseline HRV: Previous Psych Medications}

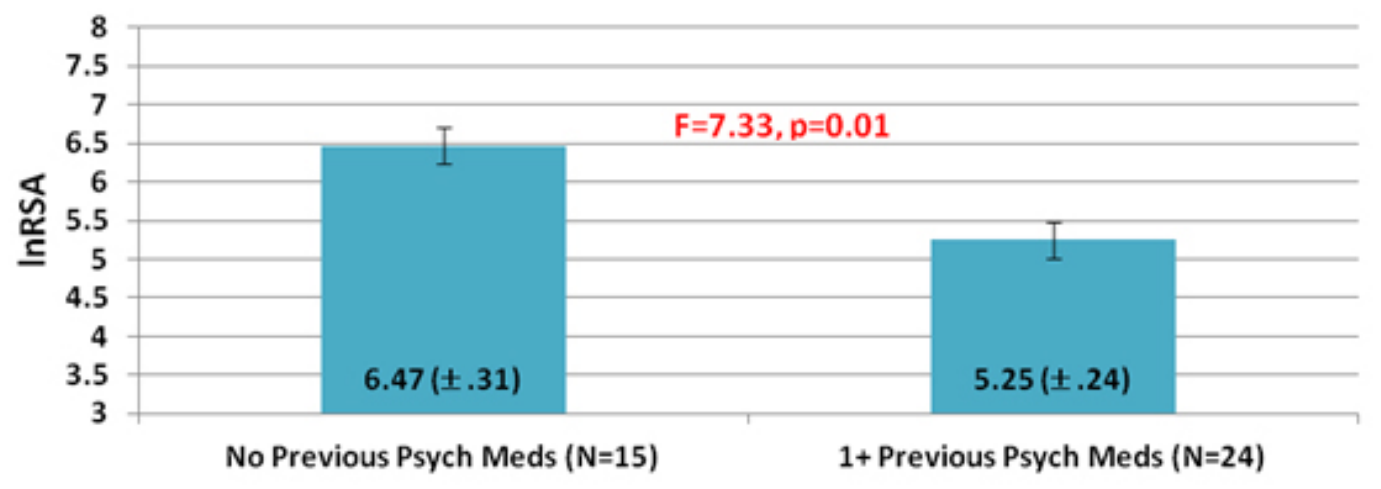

Fig. 5 Baseline HF-HRV and previous psychiatric medications. Comparison baseline HF-HRV in MDD patients with no previous psychiatric medication $(N=15)$ with MDD patients who have used at least one prior psychiatric medication $(\mathrm{N}=24)$. Baseline HF-HRV was significantly higher in medication-naïve MDD patients $(\operatorname{InRSA}=6.47, \mathrm{SEM}=0.31)$ than in MDD patients with a prior history of psychiatric medication use $(\operatorname{InRSA}=5.25$, SEM $=0.24)(F=7.33, p=0.01)$. 
The MDD group was partitioned based on occurrence of a prior major depressive episode (n = 29) or no prior major depressive episode $(n=12)$. No significant group difference in baseline RSA or LF-HRV were found between MDD patients who had a prior major depressive episode and those who had not (Data not shown).

The MDD group was partitioned based on current depressive episode length of either one to six months $(n=9)$, between six months and one year ( $n$ $=11)$, or longer than one year $(n=18)$. No significant group differences in baseline RSA or LF-HRV were found, (Data not shown).

The following inflammation biomarkers were measured: IL-1b, IL-6, IL-8, IL-10, TNF-a, MCP-1 and hsCRP. Correlational analyses were conducted comparing RSA and LF-HRV to these inflammation biomarkers. As Table 2 shows, only two significant correlations were obtained: a positive correlation between RSA and IL-1 $\mathrm{b}$ and a positive correlation between IL-10 and LF-HRV.

Table 2. Association between select inflammation biomarkers and HRV components.

\begin{tabular}{|c|c|c|c|c|c|c|c|}
\hline \multicolumn{8}{|c|}{ MDD } \\
\hline & IL-6 & IL-8 & IL-10 & TNF-a & IL-1b & MCP-1 & CRP \\
\hline RSA & $\begin{array}{l}r=-0.15 \\
p=0.29\end{array}$ & $\begin{array}{l}r=0.09 \\
p=0.55\end{array}$ & $\begin{array}{l}r=0.10 \\
p=0.50\end{array}$ & $\begin{array}{l}r=0.10 \\
p=0.49\end{array}$ & $\begin{array}{l}r=0.29 \\
p=0.05\end{array}$ & $\begin{array}{l}r=-0.19 \\
p=0.17\end{array}$ & $\begin{array}{l}r=0.12 \\
p=0.46\end{array}$ \\
\hline LF-HRV & $\begin{array}{l}r=-0.15 \\
p=0.26\end{array}$ & $\begin{array}{l}r=-0.03 \\
p=0.82\end{array}$ & $\begin{array}{l}r=0.47 \\
p<0.01\end{array}$ & $\begin{array}{l}r=0.10 \\
p=0.46\end{array}$ & $\begin{array}{l}r=0.13 \\
p=0.40\end{array}$ & $\begin{array}{l}r=-0.21 \\
p=0.12\end{array}$ & $\begin{array}{l}r=0.05 \\
p=0.75\end{array}$ \\
\hline
\end{tabular}

\section{DISCUSSION}

The two components of HRV were highly correlated (i.e., $r=0.70$ ) and behaved similarly in this study. Since there is extensive literature documenting that RSA is a robust index of cardiac vagal tone (Lewis et al., 2011), the discussion will focus on RSA. There was no significant difference in baseline RSA or LF-HRV between MDD patients and HC subjects. Earlier reports had indicated that MDD might be associated with decreased HRV, and that there is no SSRI effect on either component of HRV over a 3-6 week treatment period ${ }^{[37]}$. The study by Licht et al. (2010) may shed light on the issue whether untreated depression is associated with diminished HRV. These investigators compared continuous nonusers of antidepressants with subjects who started the use of a tricyclic antidepressant or a serotonergic and noradrenergic antidepressant and who at the two-year follow-up showed a significantly greater increase in heart rate and a decrease of RSA. Subjects who were placed on SSRI's also showed a decrease in RSA, but their heart rate did not increase. Discontinuing antidepressants systematically caused opposite effects; levels returned in the direction of those observed among nonusers. The authors concluded that this two-year longitudinal study indicated that all antidepressants cause a decrease in cardiac vagal control. After discontinuing antidepressants, autonomic function recovers, suggesting that this unfavorable effect is (partly) reversible ${ }^{[38]}$. More recent evidence indicates that tricyclic antidepressants (TCAs) are the most powerful agents in reducing HRV, followed by serotonin / norepinephrine reuptake inhibitors (SNRIs), ostensibly due to their enhancement of noradrenergic activity ${ }^{[39,40]}$. Selective serotonin reuptake inhibitors (SSRIs) have the least effect on cardiac function and may even decrease cardiac sympathetic tone ${ }^{[40]}$. Our finding that baseline RSA for patients with a history of prior use of psychiatric medication use was significantly lower than patients with no prior psychiatric medication use is therefore in agreement with the Licht et al. ${ }^{[40]}$ study, especially since we only required a four-week washout from a previous antidepressant. By contrast, our finding that neither drug treatment led to a change in RSA at the end of twelve weeks of treatment is in agreement with the Kemp et al. ${ }^{[37]}$ study and may indicate that a longer exposure to an antidepressant agent may 
be necessary to detect a change in RSA. Based on these considerations, we submit it will be critical in future studies of HRV and depression to take a detailed history of prior exposure to any specific antidepressant agent and record the length of both exposure as well as the time interval following discontinuation of the last agent prior to measuring HRV parameters.

In regards to the current study, it is also noteworthy that the patient and healthy control groups differed significantly in regards to key variables related to $\mathrm{HRV}$, such as BMI and ethnic background. Although these variables were controlled for in the analyses conducted, future studies should consider more homogenous treatment-control groups.

We determined that patients who responded to antidepressant treatment had a significantly higher RSA at baseline than those who did not respond. This pattern was mirrored in each treatment group whether receiving Escitalopram or Quetiapine. Equally intriguing is our finding that patients who responded to either antidepressant agent had a higher baseline RSA than control subjects, a group that conversely had a higher baseline RSA than treatment non-responders. This finding supports and expands the findings of Jain et al. ${ }^{[41]}$. These investigators reported a significantly higher pretreatment RSA in treatment responders prior to adjustment for covariates, but statistical significance was lost after controlling for age ${ }^{[41]}$.

Why would a high baseline parasympathetic tone predispose a MDD patient to respond favorably to antidepressant drug treatment? We believe the stress-related disruption in ANS homeostasis leads to a sustained shift in this critical balance in favor of sympathetic overdrive. It is widely accepted that depression is the stress related psychiatric disorder par excellence and genetically and epigenetically determined stress resilience and stress susceptibility play a critical role. Thus, the degree and chronicity of disruption in ANS homeostasis in depression, in which vagal regulation plays a major role, appears to be the tipping point with greater or lesser diminution in parasympathetic tone.

The critical link here is that diminution in vagal tone likely leads to disinhibition of the body's inflammatory response mediated, in part, by the Cholinergic Anti-inflammatory Pathway (CAIP) ${ }^{[5]}$. Efferent vagal fibers originating in the dorsal motor nucleus can modulate the release of inflammatory mediators, such as TNFa and IL-6, from macrophages thereby preventing over-activation of the inflammatory process without inducing immunosuppression ${ }^{[6]}$. The pro-inflammatory status associated with MDD has been adequately described in the literature and investigators have postulated that a sustained pro-inflammatory status in depression contributes to treatment resistance ${ }^{[42]}$. Our research team has measured both inflammation biomarkers and kynurenine pathway metabolites in both studies (Study-1 and Study-2) and the results were confirmatory. The results from Study-1 have been published ${ }^{[27]}$. The results from Study-2 are in preparation. Additionally, sustained low-grade inflammation in depression leads to enhancement of the kynurenine pathway of tryptophan metabolism via stimulation of indoleamine 2, 3 dioxygenase (IDO), a key enzyme responsible for the kynurenine pathway of tryptophan metabolism ${ }^{[43]}$. As a result of this metabolic diversion of tryptophan, a metabolic product, kynurenic acid, antagonizes nicotinic cholinergic transmission.

The association between HRV, specifically vagal tone as reflected in RSA and likely LF-HRV as well, and inflammation has been reported in many studies. While this was not the primary goal of this study, we feel we should make brief mention of this relatively recent area of scientific endeavor. As intriguing as the reported findings are, further investigations into this connection are warranted. We will limit this discussion to just a few representative studies. Using a stress-inducing paradigm (speech stressor), Woody et al. (2017) detected greater reductions in HF-HRV in association with the proinflammatory cytokines, IL-6 and TNFa, but not C-reactive protein (CRP) ${ }^{[44]}$. Using data from a large study (MIDUS II), Cooper et al. (2015) found LF-HRV to be inversely associated with CRP and IL-6 while HF-HRV was inversely associated with fibrinogen and CRP ${ }^{[45]}$. They concluded that their results support the existence of the vagal antiinflammatory pathway and HRV predicts levels on inflammatory biomarkers. In middle-aged men, Lampert et al. (2008) determined a graded inverse relationship between all HRV parameters (except HF) and CRP and IL-6 ${ }^{[46]}$. After controlling for confounding variables, they established that LF and VLF remained significant predictors of CRP. In cardiovascular disease, Haensel et al. (2008) determined that HRV is inversely correlated with inflammatory biomarkers both in healthy individuals and in those with cardiovascular disease ${ }^{[47]}$. In our study we found two statistically significant correlations. The positive correlation between LFHRV and IL-10 is very interesting in as much as $\mathrm{IL}-10$ is generally known as an anti-inflammatory cytokine that exerts a plethora of immunomodulatory functions during an inflammatory response and is particularly important during the resolution phase ${ }^{[48]}$. Therefore, the positive correlation with LF-HRV may be viewed as prognostically favorable. It is less clear how the positive correlation between IL1- $\beta$ 
and RSA should be understood, since this cytokine is generally viewed as being pro-inflammatory, although it has been described to exert pleiotropic effects in the brain including neuronal proliferation and differentiation ${ }^{[49]}$.

In summary, the complex interplay between brain, ANS, and the immune system ostensibly results in varying degrees of ANS disruption resulting in greater or lesser degree of parasympathetic tone diminution. If parasympathetic tone can be maintained at healthy control levels, reflecting greater ANS resilience and a lower or absent degree of inflammatory response, and therefore lesser activation of the kynurenine pathway, antidepressant drug action may proceed unhindered ultimately leading to remission. Taken together, we propose that RSA, which is a reliable measurement of parasympathetic tone, can be a useful clinical tool to predict antidepressant treatment response in MDD patients.

While the significance of the relationship between baseline HRV and treatment response is of scientific value, it will require additional research to establish its clinical significance. A main goal will be to demonstrate at what point an individual can be reliably categorized as having low or high HRV. While this study and many others have demonstrated appropriate methodological approaches to establish relationships involving HRV in the populations being studied, it will be an even greater accomplishment when an ambulatory patient can undergo a 15 minute HRV test, and have their physician explain their health risks based on their HRV score. Munoz et al. ${ }^{[50]}$ have recently shown that 2-minute ECG recordings are as accurate reflections of $\mathrm{HRV}$ as 15-30 minute measurements. The availability of an easily accessible, non-invasive and affordable test that can predict antidepressant response would be a major breakthrough. Having numerous ways to measure HRV ${ }^{[51]}$ each with their supporters and detractors, only serves to provide further challenges to the clinical application of HRV measurements.

Taken together, our study suggests that having high baseline HRV parameters (i.e., RSA and LFHRV) predisposes a patient to have a successful outcome when treated with antidepressant medication, even with an atypical antipsychotic exerting antidepressant efficacy. Vagal nerve stimulation (VNS) in conjunction with treatment as usual has been shown to have more of an antidepressant effect than treatment as usual alone in treatment-resistant depression ${ }^{[52]}$. Actually, one study found that transcutaneous VNS led to a significant increase in HRV and decrease in sympathetic tone in healthy individuals ${ }^{[53]}$. Baseline
HRV could therefore be used as a parameter in choosing which type of treatment should be appropriate for a given MDD patient.

A reliable diagnostic distinction between the depressive phase of bipolar disorder and MDD often poses serious challenges to the clinician ${ }^{[54]}$. Indeed, one recent study compared patients with bipolar II depression and patients with unipolar depression and found significantly lower RSA in bipolar II depressed patients as compared to unipolar depressed patients [55]. Similarly, HRV measurements could lead to profiling any subject with depression and predicting their degree of response to antidepressant therapy. Once the possible effects of pharmacologic agents on HRV domains have been fully clarified, wide utility of $\mathrm{HRV}$ as a biomarker will be justified.

One limitation to our study is the small sample size. Several of our trending variables could have reached significance with a larger patient and/or healthy control population. Specifically, given the small heterogeneous sub-groups (age, ethnicity, $\mathrm{BMI}$ ), it may not be viable within this sample to investigate whether baseline RSA is related to current episode length and/or previous episodes. Although this does not detract from the importance of our findings, reproducibility with a larger population would be necessary to confirm those findings that did not reach statistical significance. A larger sample size as well would increase statistical power and make any future findings to be even more robust in regards to scientific and clinical relevance. While we attempted to control for external or environmental covariables that have been discussed in the literature in conjunction with HRV, a greater sample size would allow us to control for more complex variables that future literature may also determine to influence HRV.

As in any study relying on the collection of data over time, several limitations are to be noted. Current mood state of the patient could have influenced the assigned scores in both the selfassessment and rater-administered scales. To minimize such effects, consistent provider-patient pairings were kept over the course of the study to minimize inter-observer bias and to allow the patient to receive a consistent level of care. Lastly, patients free of any psychotropic medication for at least six months preceding study enrollment and receiving no other concomitant psychotropic medication during study duration may shed light on why no significant difference was detected in our study between MDD and $\mathrm{HC}$ subjects. In this context, the use of sedativehypnotic medication is a variable that needs to be controlled for as there are reports in the literature indicating suppression of HRV by such agents 
although not all reports agree ${ }^{[56,57]}$. At the same time, observing patients on monotherapeutic psychotropic medication for a longer duration (i.e., at least 6 months) may also reveal differences between MDD and $\mathrm{HC}$ subjects.

\section{ACKNOWLEDGEMENTS}

This work was supported in part by an intramural research grant from Loyola University Stritch School of Medicine and by an investigator-initiated grant from Astra Zeneca, both awarded to Dr. Angelos Halaris.

\section{REFERENCES}

1. National Vital Statistics System. Depression. 2011; Natl Vital Stat Rep.

2. Greenberg P, Fournier A, Sisitsky T, Pike C, Kessler R. The Economic Burden of Adults with Major depressive Disorder in the United States (2005 and 2010). J Clin Psychiatry. 2015; 76: 155-162.

3. Sgoifo A, Carnevali L, Alfonso L, Amore M. Autonomic dysfunction and heart rate variability in depression. Stress. 2015; 18(3): 343-352.

4. Halaris A. Inflammation-Associated Co-morbidity Between Depression and Cardiovascular Disease. Curr Top Behav Neurosci. 2017; 31: 45-70.

5. Tracey K. Physiology and immunology of the cholinergic anti-inflammatory pathway. J Clin Invest. 2007; 117(2): 289-296.

6. Pavlov V, Tracey K. The cholinergic antiinflammatory pathway. Brain Behav Immun. 2009; 19: 493-499.

7. Alvares, GA, Quintana DS, Hickie IB, Guastella AJ. Autonomic nervous system dysfunction in psychiatric disorders and the impact of psychotropic medications: a systematic review and meta-analysis. J Psychiatry Neurosci. 2016 Mar; 41(2): 89-104.

8. Shinba T. Major depressive disorder and generalized anxiety disorder show different autonomic dysregulations revealed by heartrate variability analysis in first-onset drug-naïve patients without co-morbidity. Psychiat Clin Neurosci. 2017; 71: 135-145.

\section{DISCLOSURE STATEMENT}

The authors declare no conflict of interest.

\section{AUTHOR CONTRIBUTIONS}

Dr. Halaris designed the study, wrote the protocol, and oversaw the preparation of the manuscript. Brandon Hage actively participated in data collection, data analyses, literature search, and preparation of all drafts of the manuscript. Dr. Sinacore and Dr. Heilman performed data analyses. Dr. Porges oversaw data analyses contributed to manuscript preparation, and acted as a consultant to the coinvestigators.

9. Bassett D, Bear N, Nut D, Hood S, Bassett $S$, Hans D. Reduced heart rate variability in recurrent bipolar disorder and recurrent depression. Aust N Z J Psychiatry. 2016 Aug; 50(8): 793-804.

10. Heathers J. Everything Hertz: Methodological issues in short-term frequency-domain HRV. Front Physiol. 2014; 5: 177.

11. Denver JW. Reed SF, Porges SW. Methodological issues in the quantification of respiratory sinus arrhythmia. Biol Psychol. 2007; 286-294.

12. Akselrod S, Gordon D, Ubel F, Shannon D, Berger A, Cohen R. Power spectrum analysis of heart rate fluctuation: a quantitative probe of beat-to-beat cardiovascular control. Science. 1981; 213: 220-222.

13. Kamath M, Fallen E. Power spectral analysis of heart rate variability: a noninvasive signature of cardiac autonomic function. Crit Rev Biom Eng. 1996; 21: 245-311.

14. Goldstein D, Bentho O, Park M, Sharabi Y. LF power of heart rate variability is not a measure of cardiac sympathetic tone but may be a measure of modulation of cardiac autonomic outflows by baroreflexes. Exp Physiol. 2011; 96: 1255-1261.

15. Reyes del Paso G, Langewitz W, Mulder L, Roon A, Duschek S. The utility of low frequency heart rate variability as an index of sympathetic cardiac tone: A review with emphasis on a reanalysis of previous studies. Psychophysiology. 2013; 50: 477-487.

16. Moak J, Goldstein D, Eldadah BA, Saleem 
A, Holmes C, Pechnik S et al. Supine lowfrequency power of heart rate variability reflects baroreflex function, not cardiac sympathetic innervation. Heart Rhythm. 2007; 4: 1523-1529.

17. Pagani M, Lucini D, Porta A. Sympathovagal balance from heart rate variability: time for a second round? Exp Physiol. 2012; 97: 11411142.

18. Porges S. The polyvagal perspective. Biol Psychol. 2007; 74(2): 116-143.

19. Lewis G. Furman, S, McCool M, Porges S. Statistical strategies to quantify respiratory sinus arrhythmia: Are commonly used metrics equivalent? Biol Psychol. 2012; 89: 349-364.

20. Billman $G$. The LF/HF ratio does not accurately measure cardiac sympatho-vagal balance. Front Physiol. 2013; 4: 26.

21. Friedman B, Thayer J. Autonomic balance revisited: Panic anxiety and heart rate variability. J Psychosom Res. 1998; 44(1): 133-151.

22. Henry B, Minassian A, Paulus M, Geyer M, Perry W. Heart rate variability in bipolar mania and schizophrenia. J Psychiatr Res. 2010; 44(3): 168-176.

23. Davydov D, Shapiro D, Cook I, Goldstein I. Baroreflex mechanisms in major depression. Prog Neuropsychopharmacol Biol Psychiatry. 2007; 30: 164-177.

24. Rottenberg J, Wilhelm F, Gross J, Gotlib I. Respiratory sinus arrhythmia as a predictor of outcome in major depressive disorder. J Affect Disord. 2002; 71: 265-272.

25. Wilson ST, Chesin M, Fertuck E, Keilp J, Brodsky B, Mann JJ, et al. Heart rate variability and suicidal behavior. Psychiat Res. 2016; 240: 241247.

26.Gonzalez J, Schreck E, Batchelder A. Hamilton Rating Scale for Depression (HAM-D). Encyclopedia of Behavioral Medicine. 2013; 887-888.

27. Halaris A, Myint A, Savant V, Meresh E, Lim E, Guilemin G, Hoppensteadt D, Fareed J, Sinacore J. Does escitalopram reduce neurotoxicity in major depression? J Psychiatr Res. 2015 Jul-Aug; 66-67:118-126.

28. Sinnreich R, Kark J, Friedlander Y, Sapoznikov $D$, Luria M. Five minute recordings of heart rate variability for population studies: Repeatability and age-sex characteristics. Heart. 1998; 80: 156-162.

29. Tarkiainen TH, Timonen KL, Tiittanen $P$, Hartikainen JE, Pekkanen J, Hoek G, Ibald-Mulli
A, Vanninen EJ. Stability over time of short-term heart rate variability. Clin Auton Res. 2005; 15 : 394-399.

30. CardioEdit software. Brain-Body Center, University of Illinois at Chicago. 2007.

31. CardioBatch software. Brain-Body Center, University of Illinois at Chicago. 2007.

32. Porges S. Method and Apparatus for Evaluating Rhythmic Oscillations in Aperiodic Physiological Response Systems. Patent Number: 4,510,944. Washington DC: U.S. Patent Office. April 16, 1985.

33. Riniolo T, Porges S. Evaluating group distributional characteristics: Why psychophysiologists should be interested in qualitative departures from the normal distribution. Psychophysiology. 2000; 37: 21-28.

34. Voss A, Schroeder R, Heitmann A, Peters A, Perz S. Short-term heart rate variabilityinfluence of gender and age in healthy subjects. PLoS One. 2015; 10(3): 1-33.

35. Zhang J. Effect of age and sex on heart rate variability in healthy subjects. J Manipulative Physiol Ther. 2007; 30(5): 374-379.

36. Lenox-Smith A, Martinez J, Perahia D, Dowsett SA, Dennehy EB, Lopez-Romero P, Demyttenaere K. Treatment and outcomes for patients with depression who are partial responders to SSRI treatment: Post-hoc analysis findings from the FINDER European observational study. J Affect Disord. 2014; 169: 149-156.

37. Kemp A, Quintana D, Gray M, Felmingham K, Brown K, Gatt J. Impact of Depression and Antidepressant Treatment on Heart Rate Variability: A Review and Meta-Analysis. Biol Psychiatry. 2010; 67: 1067-1074.

38. Licht C, Geus E, Dyck R, Penninx B. Longitudinal evidence for unfavorable effects of antidepressants on heart rate variability. Biol Psychiatry. 2010; 68: 861-868.

39. Kemp AH, Brunoni AR, Santos IS, Nunes MA, Dantas EM, Carvalho de Figueiredo $\mathrm{R}$, Pereira AC, Ribeiro AL, Mill JG, Andreão RV, Thayer JF, Benseñor IM, Lotufo PA. Effects of depression, anxiety, comorbidity, and antidepressants on resting-state heart rate and its variability: An ELSA-Brasil Cohort Baseline Study. Am J Psychiatry. 2014; 171: 1328-1334.

40. Licht C, Penninx B, de Geus E. Effects of Antidepressants, but not Psychopathology, on Cardiac Sympathetic Control: A Longitudinal Study. Neuropsychopharmacology. 2012; 37 : 
2487-2495.

41. Jain F, Cook I, Leuchter A, Hunter AM, Davydov DM, Ottaviani C, Tartter M, Crump C, Shapiro D. Heart rate variability and treatment outcome in major depression: A pilot study. Int $\mathrm{J}$ Psychophysiol. 2014; 93: 204-210.

42. Boorman E, Romano G, Russell A, Mondelli V, Pariante C. Are Mood and Anxiety Disorders Inflammatory Diseases? Psychiatr Ann. 2015; 45(5): 240-248.

43. Myint A. Kynurenines: from the perspective of major psychiatric disorders. FEBS J. 2012; 279: 1375-1385.

44. Woody A, Figueroa WS, Benencia F, Zoccola PM. Stress-induced parasympathetic control and its association with inflammatory reactivity. Psychosom. Med. 2017; 79(3): 306-310.

45. Cooper TM, McKinley PS, Seeman TE, Choo TH, Lee S, Sloan RP. Heart rate variability predicts levels of inflammatory markers: Evidence for the vagal anti-inflammatory pathway, Brain Behav Immun. 2015; 49: 94-100,

46. Lampert R, Bremner JD, Su S, Miller A, Le F, Cheema F, Goldberg J, Vaccarino V. Decreased heart rate variability is associated with higher levels of inflammation in middle-aged men. Am Heart J. 2008; 156(4): e1-7.

47. Haensel A, Mills PJ, Nelesen RA, Ziegler MG, Dimsdale JE. The relationship between heart rate variability and inflammatory markers in cardiovascular disease. Psychoneuroendocrinology 2008; 33(10): 13051312,

48. Garcia JM, Stillings SA, Leclerk JL, Phillips $\mathrm{H}$, Edwards NJ, Robicsek SA, Hoh BL, Blackburn $\mathrm{S}$, Doré S. Role of interleukin-10 in acute brain injury. Front Neurol. 2017; 8: 244.

49. Tsai S-J. Effects of interleukin-1beta polymorphisms on brain function and behavior in healthy and psychiatric disease conditions. Cytokine and Growth Factors Reviews (2017).
50. Munoz M, Roon A, Riese H, Thio C, Oostenbroek E, Westrik I, de Geus EJ, Gansevoort R, Lefrandt J, Nolte IM, Snieder H. Validity of (Ultra-) Short Recordings for Heart Rate Variability Measurements. PLoS One. 2015; 10(9): e0138921.

51. Billman G. Heart rate variability - a historical perspective. Front Physiol. 2011; 2(86): 1-10.

52. Berry S, Broglio K, Bunker M, Jayewardene A, Olin B, Rush J. A patient-level meta-analysis of studies evaluating vagus nerve stimulation therapy for treatment-resistant depression. Med Devices (Auckl). 2013; 6: 17-35.

53. Clancy J, Mary D, Witte K, Greenwood J, Deuchars S, Deuchars J. Non-invasive vagus nerve stimulation in healthy humans reduces sympathetic nerve activity. Brain Stimul. 2014; 7(6): 871-877.

54. Pendergast LL, Youngstrom EAG, Merkitch K, Moore KA, Black CL, Abramson LY, Alloy LB. Differentiating bipolar disorder from unipolar depression and ADHD: The utility of the General Behavior Inventory. Psychol Assess. 2014; 26(1): 195-206.

55. Chang $\mathrm{H}$, Chang $\mathrm{C}$, Kuo $\mathrm{T}$, Huang $\mathrm{S}$. Distinguishing bipolar II depression from unipolar major depressive disorder: Differences in heart rate variability. World J Biol Psychiatry 2015; 16: 351-360.

56. Agelink MW, Majewski TB, Andrich J, MueckWeymann M. Shot-term effects of intravenous benzodiazepines on autonomic neurocardiac regulation in humans: a comparison between midazolam, diazepam, and lorazepam. Crit Care Med. 2002; 30(5): 997-1006.

57. Chang CC, Tzeng NS, Yeh CB, Kuo TBJ, Huang SY, Chang HA. Effects of depression and melatonergic antidepressant treatment alone and in combination with sedative-hypnotics on heart rate variability: implications for cardiovascular risk. World J Biol Psychiatry. 2017 Mar; 10: 1-11. 